\title{
Risk factors for development of melanoma brain metastasis and disease progression: a single-center retrospective analysis
}

\author{
Laura J. Gardner ${ }^{1,{ }^{*},}$ Morgan Ward ${ }^{6,{ }^{*}}$, Robert H.I. Andtbacka ${ }^{2,6}$, Kenneth M. Boucher ${ }^{3,6}$, Glen \\ M. Bowen ${ }^{1,6}$, Tawnya L. Bowles ${ }^{2}$, Adam L. Cohen ${ }^{3,6}$, Kenneth Grossmann ${ }^{3,6}$, Ying J. \\ Hitchcock $^{4,6}$, Sheri L. Holmen ${ }^{2,6}$, John Hyngstrom ${ }^{2,6}$, Hung Khong ${ }^{3,6}$, Martin McMahon ${ }^{1,6}$, \\ Marcus M. Monroe ${ }^{2,6}$, Carolyn B. Ross ${ }^{6}$, Gita Suneja ${ }^{4,6}$, David Wada ${ }^{1,6}$, and Douglas \\ Grossman ${ }^{1,5,6}$ \\ ${ }^{1}$ Departments of Dermatology, University of Utah Health Sciences Center, Salt Lake City, Utah \\ ${ }^{2}$ Departments of Surgery, University of Utah Health Sciences Center, Salt Lake City, Utah \\ ${ }^{3}$ Departments of Medicine, University of Utah Health Sciences Center, Salt Lake City, Utah \\ ${ }^{4}$ Departments of Radiation Oncology University of Utah Health Sciences Center, Salt Lake City, \\ Utah \\ ${ }^{5}$ Departments of Oncological Sciences, University of Utah Health Sciences Center, Salt Lake City, \\ Utah \\ ${ }^{6}$ Departments of Huntsman Cancer Institute, University of Utah Health Sciences Center, Salt \\ Lake City, Utah
}

\section{Abstract}

Objective-Melanoma metastasis to the brain is associated with poor prognosis. We sought to determine patient demographics and primary tumor factors associated with development of brain metastasis (BM) and survival. We also investigated whether the BM detection setting (routine screening vs. symptomatic presentation) affected clinical outcomes.

\begin{abstract}
Methods-A database of melanoma patients seen from 1999-2015 at our institution was reviewed to identify patients that developed BM. Patients with BM were matched by initial stage with patients who did not develop BM as a control group. Patient demographics, primary tumor characteristics, and clinical outcomes were analyzed.
\end{abstract}

Results-123 patients with BM were matched by initial presenting stage to 237 patients without $\mathrm{BM}$. Characteristics of the primary melanoma tumor associated with $\mathrm{BM}$ development included location on the scalp $(\mathrm{P}=0.030)$, nodular histologic type $(\mathrm{P}=0.020)$ and Breslow depth $>4 \mathrm{~mm}$ $(\mathrm{P}=0.048)$, while location on the leg was associated with decreased $\mathrm{BM}$ risk $(\mathrm{P}=0.006)$. In patients with BM, time to first recurrence for melanomas of the scalp was significantly shorter (10.8 vs. 24.8 months, $\mathrm{p}=0.007$ ) than non-scalp head and neck tumors. Patient stage, tumor depth, nodular

Correspondence: Doug Grossman, MD, PhD, Huntsman Cancer Institute, 2000 Circle of Hope, Suite 5262, Salt Lake City, UT 84112; (801) 581-4682, doug.grossman@ hci.utah.edu.

These authors contributed equally.

The authors have no conflicts of interest to declare. 
type, and ulceration were also associated with worse clinical outcomes. There were no differences in clinical outcomes between patients whose BM were detected upon routine screening vs. upon symptomatic presentation.

Conclusions-Factors predictive of developing BM included primary scalp location, nodular type, and depth. In BM patients, scalp location, stage, tumor depth, nodular type and ulceration, but not detection setting, were associated with worse clinical outcomes.

\section{Keywords}

Melanoma; brain metastases; predictive factors; prognostic factors; scalp

\section{Introduction}

In the United States, invasive melanoma is the fifth most common type of cancer in men and the seventh most common type in women, with 76,380 new cases predicted in 2016 and increasing incidence and mortality rates [1]. After lung and breast cancer, melanoma is the most common cancer that metastasizes to the brain [2]. Approximately $10 \%$ of patients with a history of invasive melanoma will ultimately develop brain metastasis (BM) [2], which contributes significantly to melanoma-related morbidity and mortality [3,4]. Treatment of BM is difficult, and historically such patients have had an overall survival in the range of 3-6 months [4-7]. Although average survival for these patients has improved in the current era of targeted and immune therapies [2] and with advances in radiotherapy, there remains a need to enhance our understanding of the tumor- and patient-related factors that may be associated with BM development and survival.

Previous studies examining factors predictive of melanoma BM have shown an association with depth and ulceration of the primary tumor as well as location on the head and neck [5,8-10]. In this study, along with analyzing additional patient demographic and primary tumor factors that may be associated with the development of BM, our aim was to investigate the impact of BM detection setting (routine screening vs. symptomatic presentation) on clinical outcomes. We also separately examined clinical outcomes of scalp primary tumors given their unique histopathology and patterns of recurrence relative to other head and neck primary sites.

\section{Methods}

\section{Patients}

This project was approved by the University of Utah Institutional Review Board (\#76927). Our database architect (C.B.R.) initially queried our prospectively accrued Melanoma Clinical Cancer Database (approximately 5500 patients, years 1999-2015) for melanoma patients with BM who did not have multiple primary tumors and had a diagnosis of BM recorded on or after the date of the primary tumor. We identified 134 unique patients, and then excluded those with primary of: ocular melanoma (2), gastrointestinal or esophageal melanoma (4), preceding diagnosis of other malignancy (except non-melanoma skin cancer) such as lung, breast, or colon cancer (2), history of greater than one primary invasive melanoma (1), and those with calvarial metastasis but without BM (2). After these 
exclusions, a total of 123 patients with $\mathrm{BM}$ were remaining for study. A biostatistician (K.M.B.) randomly selected control subjects from the same database to obtain approximately double the number of patients without BM in each stage category. Briefly, patients were first stratified by stage, then for each stage stratum they were randomly permuted using the "sample" command in R software (version 3.2.1, Vienna, Austria). For each grouping, approximately twice the number of patients with BM were selected to serve as controls, starting at the top of the permuted list of patients. A total of 237 patients were thus identified as not having developed BM and served as the control group.

\section{Data analysis}

The accuracy of all data for all patients was confirmed by manual review of the electronic medical record. Variables for which data entries in the database and medical record were missing or not explicitly stated were considered unknown. Mitotic rate and presence of nevus precursor in the primary melanoma specimen were not analyzed because data was missing or could not be confirmed in the majority of patients with BM. Analyses comparing the patients with $\mathrm{BM}$ and the control patients without $\mathrm{BM}$ was performed using $\mathrm{R}$ software. Fisher's Exact test was used for comparisons of categorical data. Multivariate analysis was performed using a logistic regression model for including all variables that were significant on univariate analysis. Analyses of clinical outcomes in the patients with BM was performed using GraphPad Prism software (version 6.01, La Jolla, CA). Patients presenting with an event such as recurrence or BM at time zero were excluded from analyses of clinical outcomes. Log rank (Mantel-Cox) tests were used to compare survival curves. P values < 0.05 were considered statistically significant.

\section{Results}

\section{Factors associated with development of BM}

We identified 123 patients with melanoma brain metastasis (BM) and 237 stage-matched patients without BM. Their demographics are summarized in Table I. A comparison of the two cohorts confirms that they are matched for stage $(\mathrm{P}=1.00)$. There was no predilection for male sex $(\mathrm{P}=0.12)$ or family history of melanoma $(\mathrm{P}=0.14)$. Both groups were segregated into approximate quartiles by age, and patients with BM were more likely to be in the 40-49 age grouping $(\mathrm{P}=0.038)$.

The primary tumor characteristics of the two groups are summarized in Table II. Analysis of primary tumor body site revealed several significant differences. First, patients with BM were significantly more likely to have a tumor that originated on the scalp $(\mathrm{P}=0.03)$ and significantly less likely to have a tumor that originated on an extremity $(\mathrm{P}=0.006)$. On multivariate analysis, extremity location remained negatively associated with $\mathrm{BM}$ development $(\mathrm{P}=0.017)$. Second, patients with $\mathrm{BM}$ were significantly more likely to have a nodular melanoma (NM) histologic type $(\mathrm{P}=0.02)$, although global comparison of all histologic types was not significant $(\mathrm{P}=0.11)$. With respect to Breslow depth, patients with $\mathrm{BM}$ were significantly less likely to have primary tumors $<1 \mathrm{~mm}(\mathrm{P}=0.046)$ and significantly more likely to have primary tumors $>4 \mathrm{~mm}(\mathrm{P}=0.048)$ although global comparison (for trend) of depth quartiles was not significant $(\mathrm{P}=0.11)$. We found a significant correlation 
between Breslow depth and NM type ( $\mathrm{P}<2.2 \mathrm{e}-16)$, and in multivariate analysis NM type was no longer a significant predictor and tumor depth trended toward significance $(\mathrm{P}=0.058)$. No differences were observed between the two groups with respect to ulceration of the primary tumor $(\mathrm{P}=0.27)$. Finally, there was no difference in driver mutations detected, although mutation testing was not done in most patients without BM (Table II).

\section{Clinical characteristics of patients with BM}

In patients who ultimately developed BM, the most common first site of melanoma recurrence was the brain (33\%), followed by lung (23\%), skin (21\%), and lymph node (16\%). The most common cutaneous site of recurrence was head and neck, followed by trunk (Table III). Upon initial diagnosis of BM, patients tended to have multiple rather than solitary BM (56\% vs. 44\%), with most of these patients having greater than 5 BM (Table III). Neither the site of primary tumor (Fig. 1A, $\mathrm{P}=0.40$ ) nor its histologic type (Fig. 2A, $\mathrm{P}=0.96$ or 1.00 excluding "Other" category) significantly correlated with the initial presence of solitary or multiple BM. BM were more commonly detected in the context of routine screening rather than emergent symptoms (62\% vs. $38 \%$ ); of the patients who presented with symptoms leading to diagnosis of BM, most $(76 \%)$ were not undergoing routine screening (Table III). While there was not a significant correlation of location (in general) and routine screening/symptoms (Fig 1B, $\mathrm{P}=0.14$ ), patients with $\mathrm{BM}$ detected by routine screening were significantly more likely to have tumors originating on the trunk (Fig. 1B, $\mathrm{P}=0.047$ ). The distribution of nodular and superficial spreading tumors was not significantly different in patients with $\mathrm{BM}$ detected in the context of routine screening vs. emergent symptoms (Fig. $2 \mathrm{~B}, \mathrm{P}=0.26$ or 1.00 excluding "Other" category), and between male and female patients (Fig. $2 \mathrm{C}, \mathrm{P}=0.56$ or 0.74 excluding "Other" category). Finally, while there was not a significant correlation in patients who developed BM between location (in general) and their sex (Fig. 1C, $\mathrm{P}=0.079$ ), males were more likely to have tumors originating on the trunk $\mathrm{P}=0.092$ ), while an extremity was the most common site of primary tumor in females $(\mathrm{P}=0.015)$.

\section{Clinical outcomes in patients with BM}

Median follow-up periods from date of primary tumor diagnosis and date of initial BM detection were 3.79 years (range $0.17-48$ ) and 0.68 years (range $0.10-13$ ), respectively. As expected, time to first recurrence in BM patients was significantly dependent on their initial tumor stage (Supplementary Fig. 1A, P<0.001), as average recurrence-free survival was shortest for stage III patients (13.8 months), followed by stage II patients (18.6 months) and stage I patients (55.5 months). Similar trends were observed for average time to first BM (Supplementary Fig. 1B, P<0.001) and average overall survival (Supplementary Fig. 1C, $\mathrm{P}<0.001)$. Analysis of primary tumor location revealed that BM patients whose tumors originated on the skin of head and neck had shorter times to first recurrence compared to those with tumors originating on the extremities or trunk (16.1 vs. 23.8 and 37.3 months, respectively, Fig. 1D). Similar trends were seen for average time to first BM (Fig. 1E) and average overall survival (Fig. 1F), although none of these were statistically significant.

The poorer prognosis for patients with primary tumors of the head and neck was driven largely by tumors of the scalp, as patients with scalp tumors fared significantly worse than those with other head and neck tumors with respect to time to first recurrence (10.8 vs. 24.8 
months, $\mathrm{P}=0.007$, Fig. $1 \mathrm{G})$. Times to first BM (28.8 vs. 34 months, Fig. $1 \mathrm{H})$ and overall survival (24.3 vs. 42.2 months, Fig. 1I) were also shorter in those with scalp compared to other head and neck melanomas, but these differences were not statistically significant.

Patients with nodular melanomas had significantly shorter times to first recurrence (11.4 vs. 36.1 months, $\mathrm{P}=0.013$, Fig. 2D) and to first BM (25.5 vs. 52.0 months, $\mathrm{P}=0.046$, Fig. 2E) than those with superficial spreading tumors. Overall survival was also worse in patients with nodular tumors, but the difference was not statistically significant ( $\mathrm{P}=0.28$, Fig. 2F). Consistent with the stage-dependent associations with clinical outcomes, times to first recurrence ( $\mathrm{P}<0.001$, Fig. 3A) and first $\mathrm{BM}(\mathrm{P}=0.002$, Fig. 3B), and overall survival $(\mathrm{P}<0.001$, Fig. $3 \mathrm{C})$ were significantly related to depth of the primary tumor. Similarly, the presence of ulceration in the primary tumor was significantly associated with shorter time to first recurrence (12.4 vs. 43.2 months, $\mathrm{P}<0.001$, Fig. 3D), shorter time to first BM (24.4 vs. 53.6 months, $\mathrm{P}<0.001$, Fig. $3 \mathrm{E}$ ), and worse overall survival (34.6 vs. 68.0. months, $\mathrm{P}<0.001$, Fig. 3F).

Finally, we examined whether the initial detection setting of BM (i.e. in context of routine screening vs. presenting symptoms) was associated with different clinical outcomes. We found that BM patients had similar times to first recurrence, first BM, and death regardless of the detection context (Supplementary Fig. 2A-C).

\section{Discussion}

Prior retrospective studies of melanoma patients with BM examined cohorts ranging in size from 49 to 670 patients [5,6,8-12]. The rate of BM in these cohorts was approximately $10 \%$ $[5,6,8,9,11,13]$, which corresponds to the expected risk of developing BM after a diagnosis of primary cutaneous melanoma [2]. Our cohort of 123 melanoma patients with BM represented just over $2 \%$ of patients in our melanoma database; this lower percentage likely reflects the composition of our database, which contains many patients with melanoma in situ. Our analysis of melanoma patients with BM and a stage-matched group without BM revealed several factors significantly associated with the development of BM that have been described in some, but not all previous studies as discussed below. We observed positive associations of advanced Breslow depth $(>4 \mathrm{~mm}$ ) and an inverse association with primary tumor location on the leg. We also found that advanced stage, greater tumor depth, nodular type, and ulceration were associated with worse clinical outcomes. Finally, we observed no difference in clinical outcomes between patients whose BM were detected upon routine screening compared to those who presented with neurological symptoms.

Melanoma metastasis from the primary cutaneous tumor often occurs via the lymphatic system, but hematogenous spread is also possible, particularly in cases where there is recurrence following negative sentinel lymph node biopsy or complete lymph node dissection [14-16] or in early stage patients who did not get sentinel node staging and had no evidence of nodal recurrence. Whether BM occurs predominantly via hematogenous or lymphatic spread is not clear, although Jackson et al. [17] reported that for patients with resected stage III melanoma there is no lymph node feature (i.e. nodal region, nodal size, number of involved nodes, presence of extra-capsular spread) associated with the 
development of BM. We did not examine these lymph node features or whether patients receive complete lymph node dissection as variables that may be associated with BM. We found that BM development was associated with primary tumor location on the scalp and inversely associated with location on the leg. Interestingly, our findings are consistent with a recent report by Gorka et al. [11] showing that risk of BM correlated with proximity of the primary tumor to the brain. Multiple studies [6,8,9,11,13,18,19], but not all [10,17], have shown that primary tumors of the head and neck were more likely to develop BM. In our cohort, patients with primary tumor of the scalp accounted for most of the difference in clinical outcome between those with head and neck tumors and other sites, as those with head and neck tumors excluding scalp behaved similarly to those with tumors on an extremity. Similarly, Bottoni et al. [13] reported that axial sites were more likely to develop BM than peripheral ones. Kadakia et al. [18] found that the scalp subsite was associated with worse prognosis among patients with head and neck melanomas. We have previously reported that blood and lymphatic vessel density is increased in the scalp compared to torso and extremity, as indicated by CD31 and D2-40 staining, respectively [20]. Although correlations between lymphatic density and melanoma metastasis has been reported [21], the issue remains controversial [22] and a histological study of scalp melanomas found high rates of both vascular and lymphatic invasion [23]. In addition, Hung et al.[24] observed angiotropism in 14/20 primary tumors that developed BM. We could not address this issue since the presence or absence of lymphovascular invasion was not recorded in our database.

Our study confirms the correlation of BM development with advanced tumor stage, particularly including Breslow depth $>4 \mathrm{~mm}[5,6,8,11,19]$ as reported by others. We did not find that ulceration was associated with BM, as seen in two prior studies [10,17], although multiple other studies [5,6,8,9,11] reported a correlation. Curiously, Frankel et al. [10] found that thinner primary tumors were associated with BM, although we observed a lower frequency of BM in patients with tumors $<1 \mathrm{~mm}$ in depth. Although other studies [8,25] found that NM histologic type was associated with BM development, we did not see this when corrected for tumor depth. Our patients with BM were predominantly male, as seen in other studies $[8,12,17,26]$, although our stage-matched control group was similarly malepredominant. A review of multiple studies concluded that male patients with BM have a poorer prognosis [18], although we did not address that issue here. We saw a similar distribution of SSM and NM histologic types in our males and females with BM, although females had relatively fewer primary tumors on the scalp. All clinical outcomes we examined were stage-dependent, as patients with stage III tumors (followed by stage II and stage I tumors) had shorter times to first recurrence, first BM, and death. These findings are consistent with those of Bottoni et al. [13] who reported that sentinel lymph node positivity was the strongest predictor for BM development.

Several studies noted that mutational genotype of the primary tumor was associated with BM development. Jakob et al. [27] reported a higher prevalence observed in BRAF-mutant (24\%) and NRAS-mutant (23\%) patients than in "wild-type" patients (12\%), and Maxwell et al. [28] similarly found that BRAF mutations were associated with an increased risk of BM. On the other hand, Schoenewolf et al. [25] found that BRAF mutant versus wild-type tumors showed no significant differences in propensity for BM. In our cohort, we did not see a 
difference in BRAF-positivity in patients with BM, although most patients in our control group did not have mutation testing.

Our study had several limitations inherent in a retrospective analysis including potential patient selection bias and incomplete data for some variables of potential importance. Additionally, most of our patients were diagnosed and succumbed to BM prior to the advent of newer systemic therapies as noted above, and we did not examine the effect of the surgical, radiotherapeutic, or medical treatments they received. The ideal control group would be patients who developed metastatic disease at sites other than the brain. A limitation of our control group is that although these patients were matched to our BM patients by initial tumor stage, not all these patients ultimately developed metastatic disease. In fact, we were able to confirm development of stage IV disease in only 10/237 (8.4\%) of our control patients and lack of distant metastasis in only 79/237 (33.3\%); for the remainder of our control patients $(100 / 237,42.2 \%)$, we did not have recent follow-up to ascertain this information. In reviewing the literature, we note that of nine studies examining melanoma BM, four had no control group [4,12,26,29], four had a control group similarly constructed as ours [5,6,8,9], and only one had a control group with non-brain distant metastasis [10].

Approximately $12 \%$ of patients with metastatic melanoma will have asymptomatic BM [30]. Vecchio et al. [7] found that such patients without clinical symptoms experienced a statistically significant better survival than patients with clinical symptoms, although this issue was not addressed in most prior studies of melanoma BM. By contrast, we found no differences in clinical outcomes (times to first recurrence, first BM, and death) in patients where BM was first detected by routine screening compared to those who had clinical symptoms prompting an imaging study that revealed BM. While the presence of symptoms may reflect the presence of more serious brain involvement, it may also result in earlier detection and treatment. On the other hand, detection of BM on screening may lead to the appearance of improved survival due to lead time and length time biases. Thus the similar survival regardless of method of BM detection may indicate that survival is driven primarily by how treatable the systemic disease burden is. Although the implications for screening guidelines are unclear, it is important to note that while many of our patients who developed BM prior to 2010 may not have substantially benefitted from earlier detection of BM, that may not be the case in the present era with the advent of targeted and immunotherapies.

\section{Supplementary Material}

Refer to Web version on PubMed Central for supplementary material.

\section{References}

1. Siegel RL, Miller KD, Jemal A. Cancer statistics, 2016. CA Cancer J Clin. 2016; 66:7-30. [PubMed: 26742998]

2. Cohen JV, Tawbi H, Margolin KA, Amravadi R, Bosenberg M, Brastianos PK, et al. Melanoma central nervous system metastases: current approaches, challenges, and opportunities. Pigment Cell Melanoma Res. 2016; 29:627-642. [PubMed: 27615400]

3. Bordia R, Zhong H, Lee J, Weiss S, Han SW, Osman I, et al. Melanoma brain metastases: correlation of imaging features with genomic markers and patient survival. J Neurooncol. 2016 
4. Davies MA, Liu P, McIntyre S, Kim KB, Papadopoulos N, Hwu WJ, et al. Prognostic factors for survival in melanoma patients with brain metastases. Cancer. 2011; 117:1687-1696. [PubMed: 20960525]

5. Qian M, Ma MW, Fleming NH, Lackaye DJ, Hernando E, Osman I, et al. Clinicopathological characteristics at primary melanoma diagnosis as risk factors for brain metastasis. Melanoma Res. 2013; 23:461-467. [PubMed: 24165034]

6. Gumusay O, Coskun U, Akman T, Ekinci AS, Kocar M, Erceleb OB, et al. Predictive factors for the development of brain metastases in patients with malignant melanoma: a study by the Anatolian society of medical oncology. J Cancer Res Clin Oncol. 2014; 140:151-157. [PubMed: 24292400]

7. Vecchio S, Spagnolo F, Merlo DF, Signori A, Acquati M, Pronzato P, et al. The treatment of melanoma brain metastases before the advent of targeted therapies: associations between therapeutic choice, clinical symptoms and outcome with survival. Melanoma Res. 2014; 24:61-67. [PubMed: 24121190]

8. Sampson JH, Carter JH Jr, Friedman AH, Seigler HF. Demographics, prognosis, and therapy in 702 patients with brain metastases from malignant melanoma. J Neurosurg. 1998; 88:11-20. [PubMed: 9420067]

9. Zakrzewski J, Geraghty LN, Rose AE, Christos PJ, Mazumdar M, Polsky D, et al. Clinical variables and primary tumor characteristics predictive of the development of melanoma brain metastases and post-brain metastases survival. Cancer. 2011; 117:1711-1720. [PubMed: 21472718]

10. Frankel TL, Bamboat ZM, Ariyan C, Coit D, Sabel MS, Brady MS. Predicting the development of brain metastases in patients with local/regional melanoma. J Surg Oncol. 2014; 109:770-774. [PubMed: 24862924]

11. Gorka E, Fabo D, Gezsi A, Czirbesz K, Liszkay G. Distance from Primary Tumor Is the Strongest Predictor for Early Onset of Brain Metastases in Melanoma. Anticancer Res. 2016; 36:3065-3069. [PubMed: 27272828]

12. Gugger A, Barnhill RL, Seifert B, Dehler S, Moch H, Lugassy C, et al. Cutaneous Melanoma with Brain Metastasis: Report of 193 Patients with New Observations. PLoS One. 2016; 11:e0156115. [PubMed: 27213536]

13. Bottoni U, Clerico R, Paolino G, Ambrifi M, Corsetti P, Calvieri S. Predictors and survival in patients with melanoma brain metastases. Med Oncol. 2013; 30:466. [PubMed: 23377924]

14. Jones EL, Jones TS, Pearlman NW, Gao D, Stovall R, Gajdos C, et al. Long-term follow-up and survival of patients following a recurrence of melanoma after a negative sentinel lymph node biopsy result. JAMA Surg. 2013; 148:456-461. [PubMed: 23325294]

15. Davis-Malesevich MV, Goepfert R, Kubik M, Roberts DB, Myers JN, Kupferman ME. Recurrence of cutaneous melanoma of the head and neck after negative sentinel lymph node biopsy. Head Neck. 2015; 37:1116-1121. [PubMed: 24764188]

16. O'Connell EP, O'Leary DP, Fogarty K, Khan ZJ, Redmond HP. Predictors and patterns of melanoma recurrence following a negative sentinel lymph node biopsy. Melanoma Res. 2016; 26:66-70. [PubMed: 26460498]

17. Jackson JE, Burmeister BH, Burmeister EA, Foote MC, Thomas JM, Meakin JA, et al. Melanoma brain metastases: the impact of nodal disease. Clin Exp Metastasis. 2014; 31:81-85. [PubMed: 23975156]

18. Kadakia S, Chan D, Mourad M, Ducic Y. The Prognostic Value of Age, Sex, and Subsite in Cutaneous Head and Neck Melanoma: A Clinical Review of Recent Literature. Iran J Cancer Prev. 2016; 9:e5079. [PubMed: 27703647]

19. Bedikian AY, Wei C, Detry M, Kim KB, Papadopoulos NE, Hwu WJ, et al. Predictive factors for the development of brain metastasis in advanced unresectable metastatic melanoma. Am J Clin Oncol. 2011; 34:603-610. [PubMed: 21150567]

20. Duffy K, Hyde MA, Tanner B, Goldgar D, Bowen AR, Florell SR, et al. Anatomic variability in superficial blood vessel and lymphatic vessel density. J Cutan Pathol. 2010; 37:1108-1109. [PubMed: 20492081]

21. Lubach D, Berens von Rautenfeld D, Kaiser HE. The possible role of the initial lymph vessels of the skin during metastasis of malignant tumors. In Vivo. 1992; 6:443-450. [PubMed: 1520846] 
22. Lund AW, Medler TR, Leachman SA, Coussens LM. Lymphatic Vessels, Inflammation, and Immunity in Skin Cancer. Cancer Discov. 2016; 6:22-35. [PubMed: 26552413]

23. Pasquali S, Montesco MC, Ginanneschi C, Baroni G, Miracco C, Urso C, et al. Lymphatic and blood vasculature in primary cutaneous melanomas of the scalp and neck. Head Neck. 2015; 37:1596-1602. [PubMed: 24931916]

24. Hung T, Morin J, Munday WR, Mackenzie IR, Lugassy C, Barnhill RL. Angiotropism in primary cutaneous melanoma with brain metastasis: a study of 20 cases. Am J Dermatopathol. 2013; 35:650-654. [PubMed: 23392133]

25. Schoenewolf NL, Belloni B, Simcock M, Tonolla S, Vogt P, Scherrer E, et al. Clinical implications of distinct metastasizing preferences of different melanoma subtypes. Eur J Dermatol. 2014; 24:236-241. [PubMed: 24721680]

26. Ostheimer C, Bormann C, Fiedler E, Marsch W, Vordermark D. Malignant melanoma brain metastases: Treatment results and prognostic factors--a single-center retrospective study. Int J Oncol. 2015; 46:2439-2448. [PubMed: 25891163]

27. Jakob JA, Bassett RL Jr, Ng CS, Curry JL, Joseph RW, Alvarado GC, et al. NRAS mutation status is an independent prognostic factor in metastatic melanoma. Cancer. 2012; 118:4014-4023. [PubMed: 22180178]

28. Maxwell R, Garzon-Muvdi T, Lipson EJ, Sharfman WH, Bettegowda C, Redmond KJ, et al. BRAF-V600 mutational status affects recurrence patterns of melanoma brain metastasis. Int J Cancer. 2016

29. Fife KM, Colman MH, Stevens GN, Firth IC, Moon D, Shannon KF, et al. Determinants of outcome in melanoma patients with cerebral metastases. J Clin Oncol. 2004; 22:1293-1300. [PubMed: 15051777]

30. Zukauskaite R, Schmidt H, Asmussen JT, Hansen O, Bastholt L. Asymptomatic brain metastases in patients with cutaneous metastatic malignant melanoma. Melanoma Res. 2013; 23:21-26. [PubMed: 23117880] 

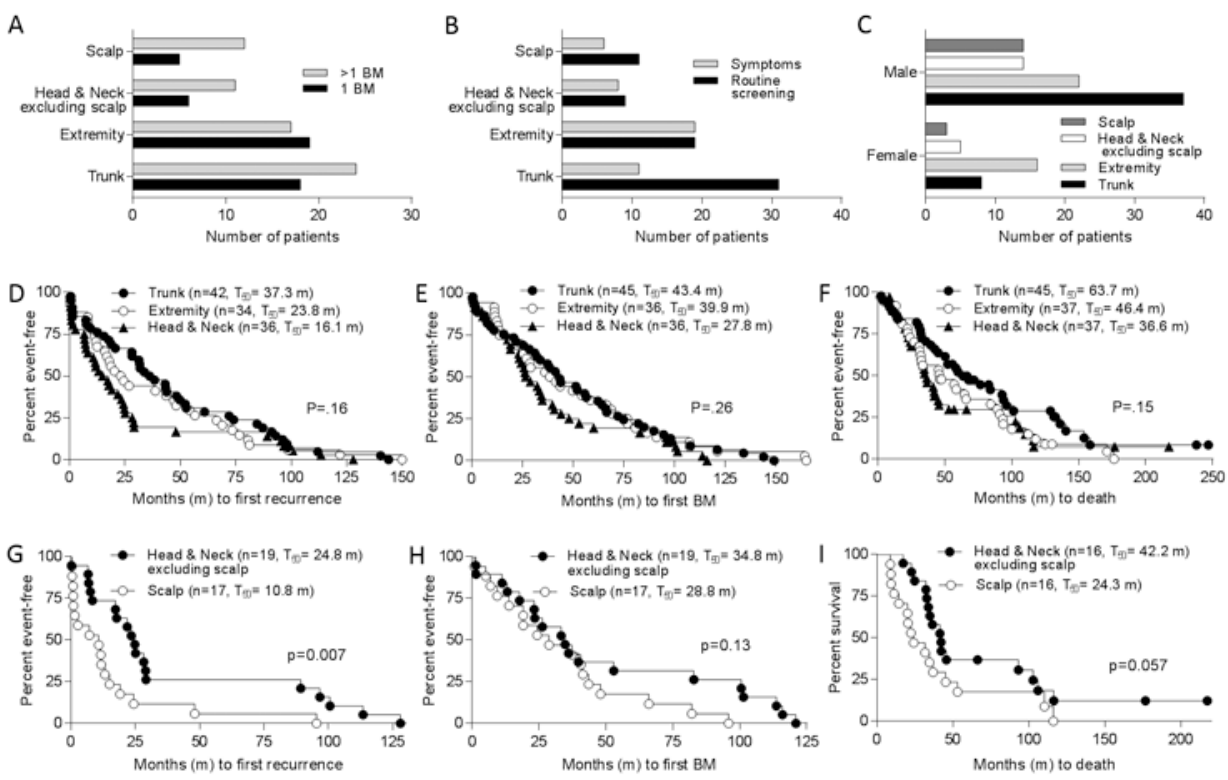

Figure 1.

Analysis of primary tumor location. A, Breakdown of primary melanoma site and number of BM detected (numerous, >1; solitary, 1). B, Breakdown of primary melanoma site and detection setting for BM (symptoms vs. routine screening). C, Distribution of primary melanoma sites by sex. Two-sided Fisher's Exact tests were used for analyses of primary site and number of BM, detection setting, and sex (see text). Patients with brain metastasis (BM) were grouped by site of primary melanoma and times (in months, $\mathrm{m}$ ) to $\mathbf{D}$, development of first recurrence; E, diagnosis of first BM; and F, death were plotted. G-I, Subset analyses of patients with primary head and neck (excluding scalp) and scalp tumors. Number of patients (n) in each group and median times to events are shown in parentheses. $\mathrm{P}$ values based on $\log$ rank tests. 
A
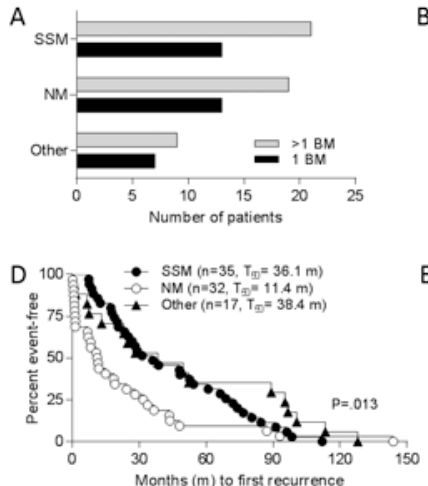

B
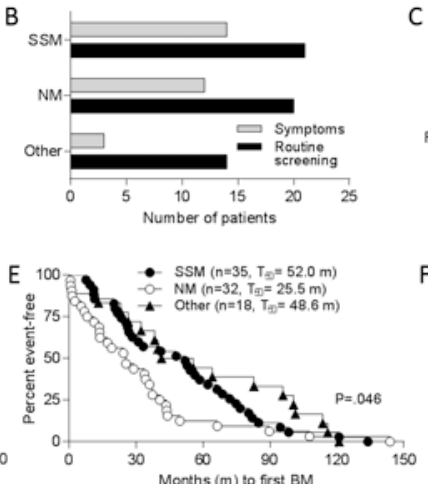

C
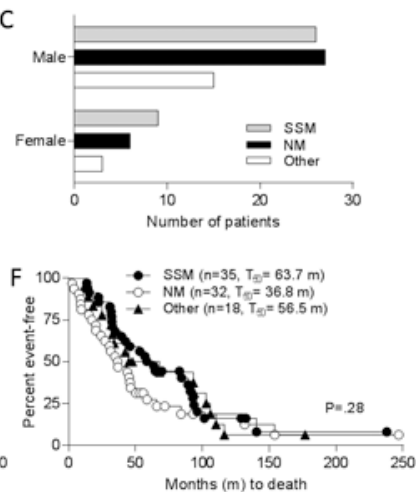

Figure 2.

Analysis of primary tumor histologic pattern. Distribution of superficial spreading (SSM) and nodular (NM) melanoma types among patients $\mathbf{A}$, with numerous (>1) or solitary (1) brain metastases (BM) detected; B, based on detection setting for BM (symptoms vs. routine screening); and $\mathbf{C}$, by patient sex. Two-sided Fisher's Exact tests were used for analyses of primary tumor histologic pattern and number of BM, detection setting, and sex (see text). Patients with BM were grouped by primary tumor histologic pattern and times (in months, m) to $\mathbf{D}$, development of first recurrence; $\mathbf{E}$, diagnosis of first BM; and $\mathbf{F}$, death were plotted. Number of patients (n) in each group and median times to events are shown in parentheses. $\mathrm{P}$ values based on log rank tests. 

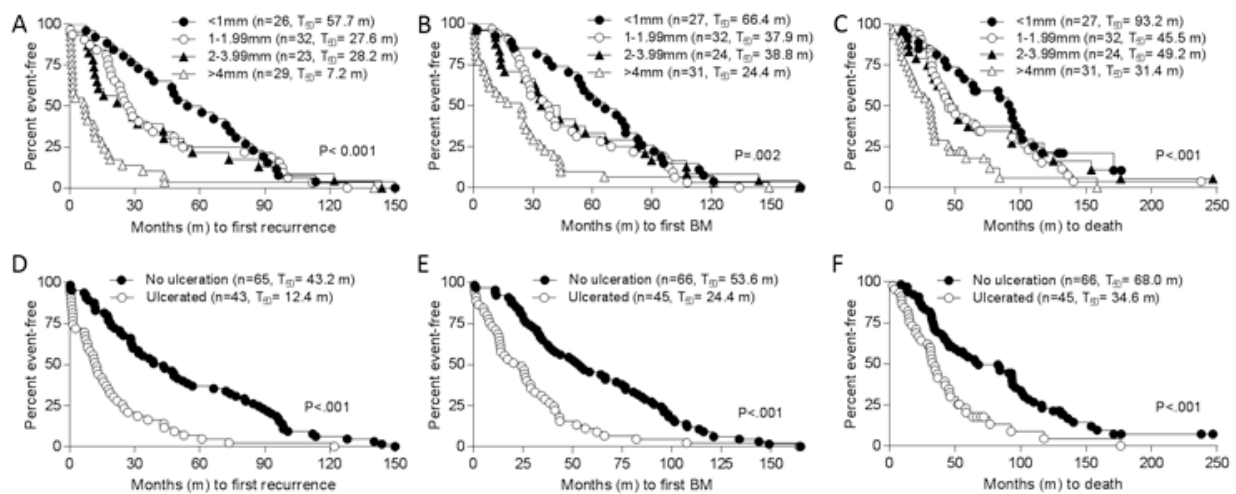

Figure 3.

Analysis of primary tumor depth and ulceration. Patients with brain metastasis (BM) were grouped by depth of primary tumors and times (in months, $\mathrm{m}$ ) were plotted to $\mathbf{A}$, development of first recurrence; $\mathbf{B}$, diagnosis of first BM; and $\mathbf{C}$, death. Patients with BM were grouped by presence or absence of ulceration in primary tumors and times (in months, $\mathrm{m}$ ) were plotted to $\mathbf{D}$, development of first recurrence; $\mathbf{E}$, diagnosis of first BM; and $\mathbf{F}$, death. Number of patients (n) in each group and median times to events are shown in parentheses. $\mathrm{P}$ values based on log rank tests. 
Patient demographics

\section{Table I}

\begin{tabular}{|c|c|c|c|}
\hline & Patients with BM & Patients without BM & $P$ value * \\
\hline Total patient number & 123 & 237 & \\
\hline Stage at initial diagnosis $\#$ & & & 1.00, global \\
\hline I & $42(34.71 \%)$ & $80(33.76 \%)$ & 0.91 \\
\hline IA & $17(14.05 \%)$ & $34(14.35 \%)$ & 1.00 \\
\hline IB & $25(20.66 \%)$ & $46(19.41 \%)$ & 0.78 \\
\hline II & $25(20.66 \%)$ & $49(20.68 \%)$ & 1.00 \\
\hline IIA & $8(6.61 \%)$ & $17(7.17 \%)$ & 1.00 \\
\hline IIB & $11(9.09 \%)$ & $22(9.28 \%)$ & 1.00 \\
\hline IIC & $6(4.96 \%)$ & $10(4.22 \%)$ & 0.79 \\
\hline III & $36(29.75 \%)$ & $70(29.54 \%)$ & 1.00 \\
\hline IIIA & $11(9.09 \%)$ & $24(10.13 \%)$ & 1.00 \\
\hline IIIB & $17(14.05 \%)$ & $30(12.66 \%)$ & 0.74 \\
\hline IIIC & $8(6.61 \%)$ & $16(6.75 \%)$ & 1.00 \\
\hline IV & $18(14.88 \%)$ & $38(16.03 \%)$ & 0.88 \\
\hline \multicolumn{4}{|l|}{ Sex } \\
\hline Male & $88(71.54 \%)$ & $137(57.81 \%)$ & 0.12 \\
\hline Female & $35(28.46 \%)$ & $100(42.19 \%)$ & 0.12 \\
\hline M:F ratio & 2.51 & 1.37 & \\
\hline Age at initial diagnosis & median 51 , range $11-86$ & median 53 , range $7-79$ & 0.043 , global \\
\hline$<40$ & $28(22.76 \%)$ & $73(30.80 \%)$ & 0.14 \\
\hline $40-49$ & $28(22.76 \%)$ & $33(13.92 \%)$ & 0.038 \\
\hline $50-59$ & $32(26.02 \%)$ & $47(19.83 \%)$ & 0.18 \\
\hline$>60$ & $35(28.46 \%)$ & $84(35.44 \%)$ & 0.20 \\
\hline \multicolumn{4}{|c|}{ Family history of melanoma ${ }^{+}$} \\
\hline Yes & $13(12.50 \%)$ & $35(20.11 \%)$ & 0.14 \\
\hline No & $91(87.50 \%)$ & $139(79.89 \%)$ & 0.14 \\
\hline
\end{tabular}

$\mathrm{BM}$, brain metastasis.

*

Fisher's Exact test

${ }^{\#}$ Stage unknown in 2 patients with BM.

${ }^{+}$Patient reported. Unknown for 19 patients with BM and 63 patients without BM. 
Table II

Primary tumor characteristics

\begin{tabular}{|c|c|c|c|c|}
\hline & Patients with BM & Patients without BM & P value* (univariate) & P value ${ }^{\#}$ (multivariate) \\
\hline Primary tumor location & & & 0.014 , global & \\
\hline Head \& Neck & $36(29.27 \%)$ & $57(24.05 \%)$ & 0.31 & \\
\hline Scalp & $17(13.82 \%)$ & $15(6.33 \%)$ & 0.030 & 0.11 \\
\hline Face & $16(13.00 \%)$ & $29(12.24 \%)$ & 0.87 & \\
\hline Neck & $3(2.44 \%)$ & $13(5.49 \%)$ & 0.28 & \\
\hline Trunk & $46(37.40 \%)$ & $75(31.65 \%)$ & 0.29 & \\
\hline Chest \& Back & $45(36.56 \%)$ & $74(31.22 \%)$ & 0.34 & \\
\hline Axilla & $1(0.81 \%)$ & $1(0.42 \%)$ & 1.00 & \\
\hline Extremity & $37(30.08 \%)$ & $103(43.46 \%)$ & 0.017 & \\
\hline Arm & $22(17.89 \%)$ & $45(18.99 \%)$ & 0.89 & \\
\hline Leg & $15(12.20 \%)$ & $58(24.47 \%)$ & 0.006 & 0.017 \\
\hline Other & $4(3.25 \%)$ & $2(0.84 \%)$ & 0.19 & \\
\hline Genital & $2(1.63 \%)$ & $2(0.84 \%)$ & 0.61 & \\
\hline Unknown primary & $2(1.63 \%)$ & $0(0.00 \%)$ & 0.12 & \\
\hline Histologic type ${ }^{+}$ & & & 0.11 , global & \\
\hline SSM & $35(40.70 \%)$ & $94(51.09 \%)$ & 0.12 & \\
\hline NM & $33(38.37 \%)$ & $44(23.91 \%)$ & 0.020 & 0.56 \\
\hline LMM & $9(10.47 \%)$ & $27(14.67 \%)$ & 0.44 & \\
\hline Other & $9(10.47 \%)$ & $19(10.33 \%)$ & 1.00 & \\
\hline Breslow depth $\neq$ & & & 0.11 , trend & 0.058, trend \\
\hline$<1.00 \mathrm{~mm}$ & $27(23.68 \%)$ & $78(34.51 \%)$ & 0.046 & \\
\hline $1.00-1.99 \mathrm{~mm}$ & $32(28.07 \%)$ & $65(28.76 \%)$ & 1.00 & \\
\hline $2.00-3.99 \mathrm{~mm}$ & $24(21.05 \%)$ & $43(19.03 \%)$ & 0.67 & \\
\hline$>4.00 \mathrm{~mm}$ & $31(27.19 \%)$ & $40(17.70 \%)$ & 0.048 & \\
\hline \multicolumn{5}{|l|}{ Ulceration \# } \\
\hline Absent & $66(59.46 \%)$ & $133(66.17 \%)$ & 0.27 & \\
\hline Present & $45(40.54 \%)$ & $68(33.83 \%)$ & 0.27 & \\
\hline \multicolumn{5}{|l|}{ Mutation testing/" } \\
\hline BRAF & $20(48.78 \%)$ & $7(50.00 \%)$ & 1.00 & \\
\hline NRAS & $7(17.07 \%)$ & $1(7.14 \%)$ & 0.66 & \\
\hline C-kit & $3(7.32 \%)$ & $0(0.00 \%)$ & 0.56 & \\
\hline No mutation detected & $11(26.83 \%)$ & $6(42.86 \%)$ & 0.32 & \\
\hline
\end{tabular}

$\mathrm{BM}$, brain metastasis.

Fisher's Exact test

\# Logistic regression model

Melanoma Res. Author manuscript; available in PMC 2018 October 01. 
${ }^{+}$Histologic type was unknown in 37 patients with BM and 53 patients without BM.

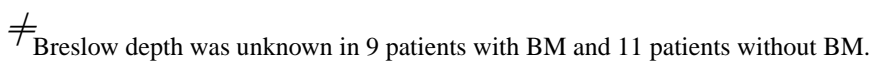

\# Ulceration was unknown in 12 patients with BM and 36 patients without BM.

/Mutation testing not done (or results unknown) 82 patients with BM and 223 patients without BM. 
Table III

\section{Clinical features of patients with BM}

\begin{tabular}{|c|c|}
\hline & \# Patients with BM \\
\hline Total patient number & 123 \\
\hline \multicolumn{2}{|l|}{ 1st recurrence site ${ }^{*}$} \\
\hline Cutaneous & $24(20.69 \%)$ \\
\hline Trunk & $5(4.31 \%)$ \\
\hline Arm & $4(3.45 \%)$ \\
\hline Face & $4(3.45 \%)$ \\
\hline Neck & $4(3.45 \%)$ \\
\hline Scalp & $3(2.59 \%)$ \\
\hline Axilla & $1(0.86 \%)$ \\
\hline Buttock & $1(0.86 \%)$ \\
\hline Groin & $1(0.86 \%)$ \\
\hline Leg & $1(0.86 \%)$ \\
\hline Brain & $27(23.28 \%)$ \\
\hline Brain + at least 1 other site & $11(9.48 \%)$ \\
\hline Lung & $27(23.28 \%)$ \\
\hline Lymph node & $18(15.52 \%)$ \\
\hline Other & $9(7.76 \%)$ \\
\hline \multicolumn{2}{|l|}{ Initial number of $\mathbf{B M}{ }^{\#}$} \\
\hline 1 & $51(43.59 \%)$ \\
\hline$>1$ & $66(56.41 \%)$ \\
\hline 2 & $16(13.68 \%)$ \\
\hline 3 & $4(3.42 \%)$ \\
\hline 4 & $1(0.85 \%)$ \\
\hline 5 & $4(3.42 \%)$ \\
\hline$>5$ & $41(35.04 \%)$ \\
\hline \multicolumn{2}{|l|}{ 1st BM detection ${ }^{+}$} \\
\hline Screening & $73(61.86 \%)$ \\
\hline Symptomatic $\neq$ & $45(38.14 \%)$ \\
\hline
\end{tabular}

BM, brain metastasis.

${ }_{1}^{*}$ st recurrence site unknown in 7 patients.

${ }^{\#}$ Initial number of BM was unknown in 6 patients.

${ }^{+}$Basis for detection unknown in 5 patients.

$\neq$ Of the screening but had emergent symptoms that prompted an unscheduled MRI or CT scan.

Melanoma Res. Author manuscript; available in PMC 2018 October 01. 